Itinéraires Itinéraires

Littérature, textes, cultures

Numéro inaugural | 2008

L'homme en tous genres

\title{
L'appropriation du masculin dans Kesso, princesse peuhle de Kesso Barry
}

Edgard Sankara

\section{OpenEdition}

Journals

Édition électronique

URL : http://journals.openedition.org/itineraires/2221

DOI : $10.4000 /$ itineraires.2221

ISSN : 2427-920X

Éditeur

Pléiade

Édition imprimée

Date de publication : 1 décembre 2008

Pagination : 163-175

ISBN : 978-2-296-07519-1

ISSN : 2100-1340

Référence électronique

Edgard Sankara, «L'appropriation du masculin dans Kesso, princesse peuhle de Kesso Barry », Itinéraires [En ligne], Numéro inaugural | 2008, mis en ligne le 01 décembre 2008, consulté le 01 mai 2019. URL : http://journals.openedition.org/itineraires/2221 ; DOI : 10.4000/itineraires.2221

\section{(C) $(\oplus \Theta$}

Itinéraires est mis à disposition selon les termes de la licence Creative Commons Attribution - Pas d'Utilisation Commerciale - Pas de Modification 4.0 International. 


\title{
L'appropriation du masculin dans Kesso, princesse peuhle de Kesso Barry
}

\begin{abstract}
This article examines how a Francophone African woman autobiographer appropriates masculine attributes in order to create a counter-discourse aimed at criticizing a male-dominated society. It also shows that the writer's conquest of masculinity is individual and does not correspond to a feminist agenda because Kesso is entangled in a series of contradictions that make her regret her choices and question her situation as an exiled African in France in an interracial marriage.
\end{abstract}

Nomades distingués dans toute l'Afrique, les Peuhls ont été l'objet de nombreuses études. L'écrivain Amadou Hampâté Bâ, peuhl lui-même, les définit comme un peuple généralement pasteur, islamisé au fil du temps et qui a su garder un fort sentiment d'identité et de noblesse ${ }^{1}$. Les peuhls sont également soumis à un code de conduite exigeant, le Pulâaku, qui fait partie de leur identité. Ainsi, chez les Peuhls pasteurs, certaines activités sont connotées comme masculines ou féminines, comme l'indique Salamatou A. Sow:

Si la vache peut être considérée comme un patrimoine identitaire commun, la conduite des troupeaux et la transformation du lait peuvent être considérés comme des patrimoines immatériaux spécifiques sur lesquels chaque groupe veille: un homme qui baratte le lait fera sourire tout le monde et une femme qui tisse la corde à veau se rend ridicule. Il y a ainsi des actes perçus comme spécifiquement féminins et d'autres comme spécifiquement masculins. La chaîne de transmission

1. A.H.Bâ, Amkoullel, l'enfant peul, Arles, Actes Sud, 1991. «Peul(e)» et «Peuhl(e)» sont les deux orthographes ramenant à la même entité. Pour des raisons d'uniformité, nous avons maintenu la même orthographe avec « $\mathrm{h}$ » que Kesso. En ce qui concerne le nom de l'autobiographe dont il sera question, nous nous référons à elle par le nom qu'elle donne en titre à son ouvrage, comme l'a fait Irène Assiba d'Almeida dans son article cité infra. 
des connaissances valorisées est une clé de compréhension du patrimoine. Chez les pasteurs peuls, la chaîne patrimoniale se construit avec un maillon masculin, symbolisé par le bâton et les pâturages, et un maillon féminin symbolisé par la calebasse et le lait. Ce dernier relève plutôt d'un matrimoine ${ }^{2}$.

Retenons ici que la société peuhle est rigidement organisée autour du masculin et du féminin, établissant ainsi un ordre des choses dont la transgression serait un sujet de ridicule ou de scandale. Kesso, l'autobiographe de notre propos, bien que peuhle, n'est pas d'une société pastorale et n'est pas non plus une femme commune, puisqu'elle est la fille de l'Almamy, roi et chef religieux d'une société peuhle sédentarisée ${ }^{3}$. Cet article se veut une analyse non pas d'un style d'écriture opposant le masculin au féminin, mais de la mise en discours d'un sujet d'énonciation, d'un « je » autobiographique portant un regard critique sur la société africaine qui l'a vue naître et grandir. Dans une société patriarcale et fortement islamisée, ce regard articule une critique autour des axes masculin/féminin, qui démontre en définitive la préférence de ce sujet féminin pour les attributs associés au genre masculin. Kesso subvertit le système de genre en montrant de l'admiration pour les femmes fortes et une volonté affichée de briser les tabous. Sa figuration thématique est celle d'une anticonformiste, une révoltée, pourtant improprement qualifiée de " révolutionnaire " par sa société parce que sa révolte n'est que singulière et ne vise pas à un changement structurel profond de son pays dans son ensemble. Cet article s'inscrit dans une socioanalyse de la société peuhle telle qu'elle est décrite par l'autobiographe, une société androcentrique, pour emprunter un terme utilisé par Pierre Bourdieu dans son ouvrage La Domination masculine 4 . La maturation du « je » féminin en quête de la possession d'attributs masculins suit une progression commençant par la lecture du masculin dans la société peuhle, l'admiration et le désir du masculin chez Kesso, et enfin son appropriation de qualités « masculines ». L'on pourrait s'interroger sur le statut de Kesso entre le masculin et le féminin: serait-elle « misovire »? D'autres questions surgissent également: le féminisme est-il un concept opératoire dans un texte écrit par une femme africaine en butte aux normes socioreligieuses antiféminines de son pays? Les nombreuses contradictions qui émaillent son texte seraient-elles révélatrices d'une limitation de son projet de liberté personnelle basée sur l'appropriation du masculin? Voici les questions qui permettront de cerner la complexité d'un texte qui s'annonce sous l'apparence de la simplicité.

2. S. A. Sow, «Le bâton de berger et la calebasse. Patrimoine et matrimoine chez les Peuls pasteurs », p. 7-8: <http://www.lemangeur-ocha.com/fileadmin/images/sciences_humaines/ SALAMATOU_SOW_caf_s_g_o.pdf $>$ (mise en ligne : avril 2006).

3. K. Barry, Kesso, princesse peuhle, Paris, Seghers, 1988.

4. P. Bourdieu, La Domination masculine, Paris, Le Seuil, 1998. 
Kesso, princesse peuhle raconte l'histoire de Kesso, née de sang royal dans la Guinée-Conakry sous la colonisation française dans les années 1940. Kesso, dont le nom signifie « vierge », affiche très tôt un esprit d'indépendance et brise les tabous socioreligieux au grand désespoir de ses parents. Au fil des années, son caractère s'affirme et, contre la coutume et contre la volonté de son père, elle se choisit un mari malléable pour éviter que lui soit imposé un époux polygame. Après son divorce d'avec son premier époux guinéen, Kesso use de toutes les stratégies pour conquérir l'homme qui deviendra son deuxième époux, l'industriel français Gérard Decoster. La deuxième partie de sa vie est passée avec celui-ci et leur fille Sandra en France, où Kesso travaille comme mannequin et accompagne son mari dans ses voyages d'affaires. Elle se trouve néanmoins dans une situation d'ambiguïté en tant que femme africaine indépendante vivant en Europe, gardant des souvenirs de son enfance partagés entre refus et nostalgie.

Au plan de la narration, l'autobiographie de Kesso se distingue par l'usage de la tradition orale comme style d'écriture. Les premiers mots du texte annoncent le récit sous cette forme:

Sandra, c'est à toi que je m'adresse. Il s'agit de ma vie et je pourrais commencer mon récit par: il était une fois dans le Fouta-Djalon une petite fille qui s'appelait Kesso. Elle était peuhle, musulmane, et princesse de sang promise à un mariage royal. Je poursuivrais un récit où l'étrange et le merveilleux tisseraient une fresque multicolore pour te faire rêver. Mais ma vie n'est pas un conte, même si elle y ressembla le temps d'une enfance. (p. 9)

D'emblée, on peut noter chez l'auteure une ambiguïté dans l'utilisation du conte pour faire le récit de sa vie, ambiguïté qui traduit à la fois un appel et une gêne. Cette gêne, chez Kesso, s'explique par la non-correspondance entre le conte qu'elle aurait souhaité raconter à sa fille et la dure réalité de la vie, qui l'a amenée à afficher une volonté d'indépendance et d'affirmation dans un contexte fortement politisé, traditionnel et islamisé. Remarquons que Kesso entend le conte dans son acception de « conte de fées ", dans le style de Cendrillon ou Blanche-Neige, où il est question de princesses; elle fait donc référence à une tradition européenne du conte, sans doute parce que la première destinataire de son texte, sa fille Sandra, n'a jamais visité l'Afrique (du moins au moment de l'écriture de cette partie de l'autobiographie). Ainsi, comme le note Irène Assiba d'Almeida: « C'est pourquoi, tel un conte, le but de l'autobiographie est non seulement de distraire mais aussi d'instruire. Destinée à Sandra, sa fille ignorante des cultures africaines, l'autobiographie de Barry se veut didactique. ${ }^{5} \gg$ En utilisant le conte comme pré(-)texte (entendu dans les deux sens), Kesso

5. I. A. d'Almeida, «Kesso Barry's Kesso, or Autobiography as a Subverted Tale», Research in African Literatures, $n^{\circ}$ 28, été 1997, numéro spécial, "Autobiography and African Literature », p. 66-82 (p. 69); nous traduisons. 
Barry fait le récit d'une vie qu'elle veut exemplaire pour sa fille, donc didactique. L'autobiographe se trouve alors dans un paradoxe postcolonial car, noble de naissance, il ne lui incombait pas de conter sa vie, c'est l'affaire des griots, gens de basse caste. Cependant, Kesso, issue de la colonisation, a en face d'elle sa fille, métisse biologique et culturelle, fruit de son mariage avec le Français Gérard Decoster. Elle doit donc conter sa vie à sa fille, étrangère aux coutumes africaines; l'autobiographe d'extraction noble fait violence à la culture traditionnelle par la force de la situation d'énonciation.

\section{La lecture du masculin et du féminin dans la société peuhle}

Enfant grandissant dans la société peuhle, Kesso apprend à lire les codes culturels qui y régissent les rapports entre les hommes et les femmes. À travers son regard observateur, l'on peut comprendre comment la société peuhle attribue des valeurs de « féminin » et de « masculin » aux hommes et femmes, aussi bien qu'à certains comportements. Nous avons signalé le faux départ du récit autobiographique: au conte de fées se substitue la dure réalité. La vie est un combat que Kesso a dû mener pour devenir la femme qu'elle voulait être dans un environnement doublement hégémonique, soumis à l'autorité de son père, le roi, et de l'islam. C'est donc en exemple qu'elle se donne à sa fille, et cet exemple semble être l'opposé de ce que fut sa propre mère pour elle. La prise de conscience de la condition particulière de la femme peuhle vis-à-vis de l'homme, Kesso l'eut très tôt en observant le rapport entre son père polygame et sa mère et ses coépouses. Kesso prend un ton de rébellion et de critique lorsqu'elle décrit l'humilité exagérée de sa mère devant son père:

\footnotetext{
Quand le matin, il sortait dans la cour et que les femmes, ses épouses, se mettaient à leur fenêtre ou devant leur porte pour essayer d'attirer son attention en espérant qu'il daignerait venir jusqu'à elles pour bavarder, je voyais ma mère, le corps tendu comme la corde d'un arc, tremblant de la tête aux pieds. Dans ces moments-là, je lisais dans son regard une telle imploration, j'y percevais une telle détresse, que j'en avais la rage au cœur. C'est certainement à cette époque que je me suis promis de ne jamais épouser un homme qui aurait plusieurs femmes. (p. 29)
}

Suite à cette scène, Kesso, outrée, conseille à sa mère de quitter son mari pour que celui-ci, conformément à la coutume, vienne la réclamer en demandant le pardon par l'offre symbolique de dix noix de cola. La réponse de sa mère est une gifle cinglante que Kesso n'oubliera pas. La promesse de ne pas épouser un homme polygame, Kesso se la fait vers l'âge de douze ou treize ans.

Dans une société musulmane et « féodale » (le mot est de Kesso) fortement hiérarchisée, Kesso apprend que le destin d'une femme est de servir l'homme et de se conformer à ses exigences. Le féminin est défini en 
termes de soumission, d'imploration et de faiblesse, dont sa mère devient pour elle l'exemple parfait. Deux femmes néanmoins détonnent dans cet environnement car, en dépit des codes sociaux, elles affirment des traits qui seraient le propre de l'homme: une certaine autorité et une forte expression d'indépendance. Ce sont ces femmes fortes qui ont du caractère à revendre que Kesso admire. Il y a l'imposante $\mathrm{M}^{\mathrm{me}}$ Diala, la griotte, qui est perçue comme "masculine » dans la communauté peuhle, comme en témoigne l'opinion du père de Kesso: «Mon père, qui la tolérait parce qu'elle le faisait rire avec ses histoires, disait d'elle que, si elle n'avait pas été femme et griotte, elle serait à coup sûr devenue un grand chef de canton. » (p. 47) La perception de la «masculinité » de $\mathrm{M}^{\text {me }}$ Diala s'aligne sur les qualités de détermination et de force. Signalons qu'à l'époque de la colonisation, le chef de canton (rôle exclusivement masculin) avait un commandement presque total sur les « indigènes ». En contrepartie, le mari de $\mathrm{M}^{\mathrm{me}}$ Diala est généralement perçu comme "féminin » par son manque de personnalité, de volonté et d'autorité. Kesso conclut par une sentence qui témoigne de tout son dédain pour cet homme: "C'était un être falot, qui vivait à l'ombre de sa femme. » (p. 46) De par cette antithèse mari-femme (M. et $\mathrm{M}^{\text {me }}$ Diala), Kesso apprend que les attributs genrés ne sont pas une question de sexe mais peuvent être transférés. Un autre exemple qui permet à Kesso de lire différemment les rapports masculin/féminin est celui de sa grand-mère paternelle. Tout comme elle respecte $\mathrm{M}^{\mathrm{me}}$ Diala, Kesso admire sa grand-mère qui symbolise, elle aussi, l'indépendance et un caractère « masculin»:

\footnotetext{
À l'inverse de ma mère, elle avait toujours eu une forte personnalité et beaucoup de caractère que le grand âge n'avait pas émoussé. On disait que, toute jeune, elle s'était battue avec un fusil aux côtés de son mari, contre sa propre famille, à un moment où les deux clans se disputaient le pouvoir. J'ai souvent entendu les griots conter cette histoire, qui me plaisait particulièrement. (p. 31-32)
}

Dans la tradition de cette société, la guerre est une activité noble réservée aux hommes qui doivent s'y illustrer par des exploits ${ }^{6}$. Qu'une femme prenne des armes tout comme son mari et se mette à combattre vaillamment, cela est vu à la fois comme une transgression dans l'ordre des choses et une démonstration de masculinité. C'est cette démonstration exceptionnelle de masculinité que Kesso admire chez sa grand-mère qu'elle considère comme "l'inverse de [s]a mère " (p. 31). Comme nous l'avons vu, Kesso réprouve la conduite soumise de sa mère, qui fait d'elle le modèle de la femme traditionnelle et musulmane absolument obéissante aux ordres de l'homme et sans pouvoir d'initiative. Une telle soumission exagérée est perçue par Kesso comme l'élément féminin à éviter: « Elle était l'exemple

6. Voir Bourdieu, op. cit., p. 41; cf. p. 86: «En fait, il n'est pas exagéré de comparer la masculinité à une noblesse. » 
parfait de la femme musulmane traditionnelle, élevée dans le respect absolu des us et coutumes, discrète jusqu'à l'effacement, obéissante jusqu'à la soumission. » (p. 27)

Kesso se rend compte également, dès son jeune âge, de la délimitation entre le monde des hommes et celui des femmes dans la répartition des espaces qui leur sont assignés; en décrivant la case à palabres, Kesso a conscience que c'est l'espace public réservé aux hommes: " C'était la case des hommes, et aucune femme n'avait le droit d'y pénétrer, excepté pour quelques tâches ménagères indispensables. L'endroit était tabou. » (p. 25) Comme le dit Bourdieu:

La force de l'ordre masculin se voit au fait qu'il se passe de justification: la vision androcentrique s'impose comme neutre et n'a pas besoin de s'énoncer dans des discours visant à la légitimer. L'ordre social fonctionne comme une immense machine symbolique tendant à ratifier la domination masculine sur laquelle il est fondé: [...] c'est la structure de l'espace, avec l'opposition entre le lieu d'assemblée ou le marché, réservés aux hommes, et la maison, réservée aux femmes, ou, à l'intérieur de celle-ci, entre la partie masculine, avec le foyer, et la partie féminine avec l'étable, l'eau et les végétaux. (p. 22-23)

L'analyse de Bourdieu de la société kabyle trouve des applications dans la société peuhle à cause des valeurs conservatrices partagées.

\section{L'admiration et le désir du masculin chez Kesso}

L'observation des rapports masculin/féminin amène Kesso à éprouver de l'admiration pour les femmes qui incarnent ou développent des traits masculins, ce qui crée en elle un désir de se les approprier, surtout par association avec la gent masculine. Plusieurs occurrences dans l'autobiographie de Kesso indiquent sa volonté de s'associer au masculin via les jeunes garçons de son âge :

Je préférais m'amuser avec les garçons plutôt qu'avec les compagnes de mon âge, que je trouvais trop sages et déjà trop soumises. Il planait audessus des jeux interdits que je partageais avec les garçons l'idée de faute. (p. 73)

On lui [son père] rapportait que je passais mon temps à jouer au ballon avec les garçons, que je portais des shorts et seulement un maillot lorsque j'allais me baigner à la rivière, que je faisais du vélo et montrais mes jambes, on lui disait encore... Que ne lui disait-on pas? Tout cela était vrai, et je ne m'en cachais pas. Je passais le plus clair de mon temps avec les garçons de Mamou, j'aimais leurs jeux violents, que je préférais à ceux des filles de mon âge, et personne n'aurait pu m'empêcher de faire ce dont j'avais envie. (p. 55-56) 
Nous étions nombreux pour ces chasses [...] et, au milieu de tous ces hommes, moi, la petite princesse incorrigible. (p. 91)

Je voulais être comme les garçons, faire comme eux, et rester digne. (p. 92)

Je préférais de beaucoup les jeux des garçons à ceux des filles. (p. 93)

Dans une société fortement hiérarchisée et où les rôles du genre sont rigidement codifiés, Kesso fait doublement figure de rebelle: premièrement, elle transgresse l'ordre masculin/féminin, dédaignant les jeux des jeunes filles et s'associant à ceux réservés aux garçons; deuxièmement, elle transgresse l'ordre politique qui veut que la fille du roi soit réservée dans sa conduite et dans son association avec les mâles. Son anticonformisme social s'exprime par un renversement de codes culturels que nous qualifierons volontiers de tactique subversive lui permettant de circonvenir les restrictions à sa liberté. Chez Kesso, cette subversion passe souvent par une transgression vestimentaire et il y a une abondance de références au travestissement de la jeune princesse, notamment la scène où elle insiste pour suivre son frère Alphadio dans une cérémonie initiatique secrète et réservée aux hommes:

À force d'être tourmenté, Alphadio, son tour venu, accepta de m'emmener avec lui; " habille-toi en garçon, et viens avec moi », me dit-il un soir. $C$ 'était un crime, évidemment, mais je n'avais peur de rien, et Alphadio non plus. Cette nuit-là, j'ai pénétré dans ce monde interdit aux femmes, et j'ai assisté aux danses guerrières et aux luttes violentes de ces garçons à peine plus âgés que moi. (p. 93 ; c'est nous qui soulignons)

Plus tard, elle dira à son autre frère Thierno Mamadou qui la reprenait sur la liberté avec laquelle elle laissait voir ses cuisses sur le manège d'une fête foraine: "- Si ce n'est que ça, la prochaine fois, je mettrai un pantalon. Thierno Mamadou ne comprenait pas ce défi: une femme musulmane ne porte pas de pantalon!» (p. 77) Le texte ne dit pas si Kesso a joint l'acte à la parole, mais le lecteur peut se faire une idée de la réalisation d'un tel projet par la petite princesse anticonformiste. Le port de vêtements masculins par une femme, dans une société patriarcale et islamisée, constitue un scandale; cela était néanmoins encouragé par l'institutrice Fanta Sylla. Celle-ci s'est attiré de vifs reproches en invitant les filles dont faisait partie Kesso à s'habiller en short pendant la séance d'éducation physique :

Les notables étaient horrifiés; ils venaient voir mon père pour lui dire: «Almamy, comment pouvez-vous tolérer que cette femme vive parmi nous? Il est impossible d'imaginer ce qu'elle exige de nos filles. Elle les déshabille à moitié, on voit leurs jambes, et elle les fait jouer au ballon comme des hommes. Quand elles tombent, elles écartent les cuisses et...» (p. 104)

Kesso affirme que l'institutrice Fanta Sylla, avec son orientation marxiste et sa détermination à lutter contre l'hégémonie des hommes sur les femmes, fut celle qui la confirma dans ce qu'elle sentait confusément en elle: 
sa préférence pour un masculin qui lui donnait un air de liberté (p. 103). Kesso confesse également sa passion pour certaines activités strictement réservée aux hommes, à commencer par la chasse. Suite à son insistance et après que son frère Thierno Mamadou l'a qualifiée de « garçon manqué », ce dernier accepte de l'emmener à la chasse à l'éléphant. Kesso exprime dans ce passage son attrait pour cette «vie extraordinaire qui [1]'attir[e] autrement plus que les palabres et les occupations casanières des femmes de la concession » (p. 89). Pendant la colonisation, le père de Kesso, souverain de sa région, devait s'assurer de la rentrée de l'impôt pour les autorités françaises. L'Almamy envoie son fils Thierno Mamadou faire la collecte de l'impôt. Encore une fois, Kesso précise : «C'était un domaine strictement réservé aux hommes, mais je m'arrangeais, une fois encore, pour me faire emmener. » (p. 95) Non contente de l'idée d'accompagner son frère, Kesso revendique, à la fin de la tournée, le droit, lorsqu'elle sera adulte, de se charger personnellement de la collecte de l'impôt. Cet affront à la coutume et à la bienséance peuhle est vite puni par sa mère, gardienne des traditions: «Lorsque je revenais à Mamou, ma mère m'attendait, et j'avais droit à une correction. Pour ma fugue et pour mon insolence, car affirmer sans honte qu'un jour je prendrai la place d'un homme était de la dernière impertinence. » (p. 98)

Après l'observation du contraste social entre « masculin » et « féminin », Kesso éprouve donc un désir de s'approprier les rôles et les symboles normalement réservés aux hommes. Toutes ses tentatives de transgression de l'ordre immuable de sa société prouvent à quel point ce désir était intense en elle, mais elles montrent également que la société androcentrique peuhle était peu disposée à accepter pareille perturbation. Les alliés masculins de Kesso, son frère Alphadio et dans une grande mesure son père, la laissent faire, mais c'est surtout avec l'espoir que « cela lui passera ». Le silence de son père devant le comportement atypique de sa fille étonne cependant et pourrait être compris comme un certain favoritisme à son égard. En effet, tout porterait à croire que le père de Kesso, décrit comme le représentant de la féodalité et de la religion ainsi que le responsable de la servitude de sa mère, s'opposerait à l'indépendance de sa fille. Au contraire, le silence de celui-ci témoigne d'une complicité tacite que Kesso paie par une admiration totale car elle reconnaît que sans son laisser-faire, elle ne serait pas ce qu'elle est devenue; la dédicace du livre est révélatrice de la gratitude de Kesso: « À l'Almamy, mon père, à Gérard mon mari, les deux hommes qui m'ont permis d'être moi-même. » (p. 7) Consciente du poids de cette société, Kesso sait que si elle s'y soumet, elle finira par devenir l'une des épouses d'un homme polygame. Elle épouse donc un homme de son ethnie, mais qui ne correspond pas aux attentes de sa famille royale. Ce mariage « contre-nature » de Kesso vise à contourner les diktats de la société androcentrique, par un renversement des codes à son profit: alors que la coutume veut que ce soient l'homme et les parents qui choisis- 
sent l'épouse, c'est Kesso elle-même qui choisit d'épouser Baïlo, fils cadet d'une modeste famille et qu'elle confesse ne pas aimer d'amour.

\section{La conquête du masculin: féminisme ou « misovirisme » ?}

La vraie conquête de la masculinité, Kesso ne l'accomplit qu'après avoir retenu l'attention de Gérard Decoster, industriel français opérant en Guinée. Kesso renverse maintenant le schéma classique du mâle faisant la cour à la femelle, car c'est elle qui prend les choses en main et décide de faire la cour à Decoster. La seconde moitié de son œuvre montre toutes les tactiques déployées par Kesso pour vaincre les réticences de Decoster: avances, recours aux pratiques magiques, course-poursuite, etc. Kesso est figurée comme un « chasseur » à la poursuite de sa « proie », figuration qui fait écho à la passion pour la chasse de l'enfance de Kesso et confirme en même temps son appropriation du masculin.

En étudiant une autobiographie écrite par une femme africaine en opposition avec sa culture d'origine, l'on peut se poser la question de savoir si l'écrivaine fait acte de féminisme. Dans l'article cité plus haut, Irène d'Almeida a montré que la conduite « subversive » de Kesso pourrait s'apparenter à un acte féministe. Or, Kesso elle-même dira, lors d'une interview donnée au magazine Amina, qu'elle n'est pas féministe ${ }^{7}$. Cependant, il est aussi possible de caractériser Kesso comme une " misovire », c'està-dire une femme qui « hait » les hommes, ou qui n'en trouve pas à son goût ${ }^{8}$. Reprenant un terme utilisé par Werewere Liking dans Elle sera de jaspe et de corail (Journal d'une misovire...), Rangira Béatrice Gallimore définit « misovire » ainsi :

La « misovire » ou plutôt le « misovirisme », si nous pouvons nous permettre ici d'en faire une idée, est né de la frustration de la femme africaine qui n'arrivait pas à trouver un homme répondant à ses aspirations au sein de l'Afrique moderne. Le « misovirisme» se distingue donc du féminisme radical et doit être appréhendé en termes dialectiques ${ }^{9}$.

En accord avec la définition de Gallimore, nous pouvons affirmer que Kesso fait figure de « misovire » dans la société peuhle où elle a grandi, car aucun des hommes qui ont cherché à la séduire n'a trouvé sa faveur. Son choix

7. A. B. Diallo, « Kesso, princesse peuhle », Amina, n 220, août 1988, p. 66.

8 . Le terme «misovire» pourrait porter à critique puisqu'il existe la possibilité d'écrire « misandre » qui rend la même idée. Cependant, nous privilégions ce néologisme forgé par Werewere Liking, qui l'utilise d'ailleurs avec son contraire dans la même phrase au début de son livre: "Et la misovire que je suis rencontrera un misogyne », Elle sera de jaspe et de corail (Journal d'une misovire...), Paris, L'Harmattan, 1983, p. 9.

9. R. B. Gallimore, «Écriture féministe? Écriture féminine? Les écrivaines francophones de l'Afrique subsaharienne face au regard du lecteur/critique ", Études françaises, n 37, 2, 2001, p. 79-98 (p. 86 et 87); <http://www.erudit.org/revue/etudfr/2001/v37/n2/009009ar.pdf $>$. 
délibéré de se marier avec Baïlo était plus un calcul que de l'amour. Cette Afrique en transition vers la modernité n'offrait pas à Kesso un homme digne d'elle, ce qui peut également expliquer son choix d'épouser ensuite un non-Africain. Ainsi, jusqu'à sa rencontre avec Gérard Decoster, Kesso fait figure de «misovire » dans l'espace africain, mais cet état cesse dès qu'elle se marie avec l'industriel français. L'ironie du parcours initiatique de Kesso est que, fille d'un royaume qui avait gardé une certaine indépendance et une hauteur envers les Français malgré le joug de la colonisation, Kesso se conjoint avec un représentant de l'occupant auparavant conspué. Un ressort de cette attraction pourrait être le fait que, puisque Baïlo ne lui offrait plus l'échappatoire escomptée contre sa société trop traditionnelle, Kesso a préféré un homme qui symbolisait la France - non pas la France des colonisateurs auxquels elle était habituée et qu'elle dédaignait, mais celle d'un énarque fraîchement débarqué et sans lien immédiat avec la situation coloniale. En ce sens, l'anticonformisme de Kesso avait besoin d'un exil et c'est Decoster qui le lui offrait. En proposant cette interprétation, nous ne voulons pas dénier qu'il y ait eu des sentiments d'amour entre les époux, mais dans l'économie du texte, Decoster apparaît comme un moyen pour Kesso de s'échapper d'une société où elle étouffe d'être une femme « masculine » en butte à la tradition.

\section{Discours contradictoire, usurpation du titre et limitations du projet}

Si l'histoire de Kesso s'arrêtait ici, il s'agirait d'une vie arrachée de haute lutte au monde des hommes par l'appropriation du masculin. Cependant, la trajectoire de Kesso révèle une série de contradictions dans son discours de puissance qui limitent sa portée de vie exemplaire. Écrivaine faisant un discours didactique à sa fille, en montrant sa préférence pour le masculin, Kesso offre des contradictions à deux niveaux lorsqu'elle parle d'un homme, Iéro, et d'une femme, Hadiatou, qu'elle admire.

Dans la société peuhle où évolue Kesso pendant la colonisation française, il existait encore des esclaves soumis à l'autorité de l'Almamy, que les nobles ne devaient pas fréquenter. Cependant, Kesso le fera et éprouvera une admiration particulière pour l'un d'eux dont elle admire la virilité et la force: «Il s'appelait Iéro, il était fort, il était beau, j'étais en admiration devant lui. Avec la seule force de ses bras, il pouvait arracher un arbre ou maîtriser un jeune taureau échappé. » (p. 39) L'admiration de Kesso pour Iéro prend des allures d'amour, sentiment que Kesso relativise devant sa fille, Sandra: « J'étais amoureuse de Iéro, non pas comme on l'entend ici, mais comme on peut aimer un beau cheval de race. » (p. 40) Le déictique spatial « ici » se réfère à la France, et Kesso nie qu'elle avait un amour passionné pour le jeune esclave, justifiant ses sentiments envers lui en les comparant à ceux que l'on pourrait avoir pour un cheval. Là où une contradiction explicite s'établit dans le discours de Kesso, c'est lorsque, après avoir déclaré à son père qu'elle voudrait épouser Iéro, son père, amusé, dé- 
cide de le lui « donner ». Et Kesso de conclure: « Aujourd'hui, je me rends compte de ce que ce mot a de terrible, car mon père me donnait Iéro comme on offre un cheval ou un objet. » (p. 40) Kesso ne semble pas se rendre compte que sa description de ses sentiments pour Iéro participe de la même attitude « terrible » qui l'offusque chez son père. On pourrait conclure que Kesso voit en Iéro des traits masculins qu'elle admire, comme la force et la détermination, portés à leur plus haut niveau. Cependant, son hésitation à homologuer sa relation à Iéro à de l'amour est en conformité avec son « misovirisme » : si Iéro est un homme extraordinaire, il n'en demeure pas moins un esclave; malgré la susceptibilité de Kesso donc, il n'était pas en mesure de lui offrir la porte de sortie désirée. Iéro était une possibilité limitée du masculin; il fallait attendre Gérard.

Il y a également une contradiction et une ironie dans le sous-titre de l'autobiographie de Kesso dans la mesure où, malgré sa naissance, sa conduite anticonformiste lui retire aux yeux de sa société tout droit au titre de princesse peuhle. La vraie princesse, c'est sa sœur Hadiatou, et cela selon l'aveu de Kesso elle-même:

Ma sœur était une vraie princesse peuhle, une jeune fille modèle, à l'image de ce que souhaitaient tous les parents. Respectueuse des traditions, elle se comportait en toutes occasions selon les vœux de ma mère. Les yeux toujours baissés lorsqu'on lui adressait la parole, elle ne parlait que quand on la questionnait et elle s'habillait décemment: jamais de robe qui laisse voir les genoux, ou de pantalon qui moule les formes; elle ne portait que le pagne et la chemise traditionnels. (p. 84)

En outre, plus sa sœur se soumet aux coutumes peuhles en restant une femme traditionnelle, plus Kesso s'occidentalise, s'éloignant par là même des valeurs de sa société. À noter que dans cette société peuhle, qui a gardé son intégrité culturelle et politique malgré la colonisation française, les Européens et leurs tenues vestimentaires étaient perçus comme ridicules:

Les jours de fête, lorsque nous mettions nos plus beaux habits, les hommes en boubou blanc ou bleu, les femmes avec leurs tuniques multicolores, et que le commandant de cercle et ses amis se présentaient dans leurs tenues ridicules, en short et en casque colonial, transpirant et soufflant comme des malheureux, nous n'avions aucune pitié pour ces hommes qui, à nos yeux, n'avaient aucune classe. (p. 129)

La contradiction de Kesso est visible lorsqu'elle se vêt à l'européenne, créant ainsi un écart entre son discours personnel et le «nous » du passage cité. Hadiatou est l'image de sa mère et à ce titre mérite le titre de princesse; logiquement, Kesso devrait éprouver une répulsion pour sa sœur qui a copié le modèle de soumission maternelle. Cependant, c'est l'inverse qui se produit et l'on pourrait suggérer qu'une fois s'être approprié le masculin et après avoir consolidé sa vie en France, Kesso a été confrontée à son intégration au monde français où elle n'était pas complètement acceptée en 
tant que Noire et épouse d'un Français. Cet inconfort existentiel l'amène donc à regretter les choix de son enfance qui lui ont permis d'avoir un tel destin, et sa sœur Hadiatou, son contraire parfait, lui apparaît comme la possibilité et le regret d'une autre vie qu'elle aurait pu mener dans une intégration totale en Afrique. L'insistance de Kesso sur le titre « princesse peuhle » en page de couverture de son autobiographie témoignerait ainsi d'un désir de s'octroyer un titre qu'elle a, en quelque sorte, refusé. Kesso, adulte, ferait ainsi l'aveu d'avoir poussé sa balle trop loin dans le camp des Blancs et de s'être occidentalisée, contrairement à sa sœur, aveu qui cacherait un certain regret de n'être pas devenue une véritable princesse peuhle (p. 36). Même l'entreprise autobiographique du « parler de moi », Kesso la perçoit comme une anomalie, « une maladie de Blanc, dont [elle est] atteinte [elle] aussi » (p. 36). Si le but de Kesso était de se donner en modèle à sa fille Sandra, ces diverses contradictions dans son texte infirment la logique de son projet. Enfin, commencée comme un dialogue avec Sandra, le texte se termine comme un monologue témoignant de la défaite de Kesso, qui n'a pas su s'intégrer dans sa société d'origine et qui se retrouve également comme une étrangère en France.

\section{Conclusion}

L'appropriation du masculin par une jeune « princesse peuhle » passe par une lecture de sa société et est guidée par un modèle à ne pas suivre, celui de sa mère, ainsi que par les exemples positifs que représentent $\mathrm{M}^{\mathrm{me}}$ Diala et sa grand-mère paternelle. Autobiographie qui se veut exemplaire, Kesso, princesse peuhle est l'histoire d'une lutte d'affirmation personnelle contre les aléas de la naissance dans une société africaine traditionnelle et islamisée, et déjà en pleine mutation sous l'effet de la colonisation française. On suit aussi la montée en puissance d'une femme qui a su dès le départ distinguer les codes culturels de sa société et qui a affirmé le désir de les contrarier par son appropriation du masculin. Kesso, « vierge », est à la fois un déshonneur et un scandale pour ses parents, autorités religieuses et coutumières, dont elle ne satisfait pas les attentes. Toute son entreprise d'écriture prolonge son désir de briser les tabous et restrictions qui entourent les relations hommes-femmes dans sa communauté d'origine. L'autobiographie de Kesso, qui se présente comme la voix d'une femme qui fait le point sur sa vie et le vécu d'une Afrique en mutation entre la colonisation et l'indépendance, soulève plusieurs questions. Prenant Sandra sa fille comme interlocutrice au début de son autobiographie, Kesso veut lui montrer par son exemple qu'une large place est accordée à la jeune fille qu'elle est; ce pseudo-dialogue d'affirmation de soi et de sa fille se termine en un véritable monologue sur la place d'une femme africaine en « exil » en France et sur le regret de la vie communautaire traditionnelle en Guinée qu'elle a laissée derrière elle. La finalité du projet de se donner en exemple à sa fille n'est pas atteinte du fait des diverses contradictions 
de l'autobiographe en rapport avec sa mère, sa sœur Hadiatou et même sa présence en France. Kesso, princesse peuhle aurait pu être aussi une contradiction pour ceux qui affirment que l'autobiographie africaine se préoccupe trop de la collectivité. Kesso Barry nous donne l'exemple d'une individualité qui s'est affirmée dans un contexte collectiviste on ne peut plus contraignant; cependant, son autobiographie qui aurait pu faire exception hésite vers la fin entre l'individualisme auquel elle a atteint et le regret d'une vie communautaire qu'elle avait rejetée; son autobiographie est un cas limite d'exception au label communautariste assigné à l'autobiographie africaine. Ce projet d'appropriation du masculin pour s'affirmer dans une société androcentrique est également limité par l'insistance que Kesso met à défendre ses propres intérêts et non pas ceux des femmes peuhles en général; ce qui lui retire toute initiative féministe, et ce qui d'emblée la projette comme rebelle et non pas comme révolutionnaire. Pour utiliser une distinction proposée par Michel de Certeau, nous pouvons dire que l'appropriation du masculin par Kesso relevait plutôt du tactique que du stratégique, la tactique étant un art du faible n'ayant pas « la possibilité de se donner un projet global ni de totaliser l'adversaire dans un projet distinct, visible et objectivable ${ }^{10} \gg$. C'est sans doute la raison pour laquelle le succès de Kesso est limité, tout comme la réception critique qui a été accordée à son œuvre.

Edgard Sankara

Université du Delaware, États-Unis

10. M. de Certeau, L'Invention du quotidien, I, Arts de faire, Paris, Gallimard, 1990, p. 61. 